\title{
Efficacy and safety of bilateral continuous theta burst stimulation (cTBS) for the treatment of chronic tinnitus: design of a three-armed randomized controlled trial Carola Arfeller ${ }^{1}$, Reinhard Vonthein ${ }^{2}$, Stefan K Plontke ${ }^{3}$ and Christian Plewnia*1
}

\begin{abstract}
Address: ${ }^{1}$ University Hospital of Tübingen, Department of Psychiatry and Psychotherapy, Osianderstraße 24, 72076 Tübingen, Germany, ${ }^{2}$ University Hospital of Tübingen, Department of Medical Biometry, Westbahnhofstraße 55, 72070 Tübingen, Germany and ${ }^{3}$ University Hospital of Tübingen, Department of Otolaryngology, Elfriede-Aulhorn-Straße 5, 72076 Tübingen, Germany
\end{abstract}

Email: Carola Arfeller - carola.arfeller@med.uni-tuebingen.de; Reinhard Vonthein - reinhard.vonthein@imbs.uni-luebeck.de; Stefan K Plontke - stefan.plontke@uni-tuebingen.de; Christian Plewnia* - christian.plewnia@uni-tuebingen.de

* Corresponding author

Published: 21 August 2009

Trials 2009, 10:74 doi:10.1186/1745-62/5-10-74
Received: 10 April 2009

Accepted: 2I August 2009

This article is available from: http://www.trialsjournal.com/content//0/I/74

(c) 2009 Arfeller et al; licensee BioMed Central Ltd.

This is an Open Access article distributed under the terms of the Creative Commons Attribution License (http://creativecommons.org/licenses/by/2.0), which permits unrestricted use, distribution, and reproduction in any medium, provided the original work is properly cited.

\begin{abstract}
Background: Tinnitus, the perception of sound and noise in absence of an auditory stimulus, has been shown to be associated with maladaptive neuronal reorganization and increased activity of the temporoparietal cortex. Transient modulation of tinnitus by repetitive transcranial magnetic stimulation (rTMS) indicated that these areas are critically involved in the pathophysiology of tinnitus and suggested new treatment strategies. However, the therapeutic efficacy of rTMS in tinnitus is still unclear, individual response is variable, and the optimal stimulation area disputable. Recently, continuous theta burst stimulation (cTBS) has been put forward as an effective rTMS protocol for the reduction of pathologically enhanced cortical excitability.

Methods: 48 patients with chronic subjective tinnitus will be included in this randomized, placebo controlled, three-arm trial. The treatment consists of two trains of cTBS applied bilaterally to the secondary auditory cortex, the temporoparietal associaction cortex, or to the lower occiput (sham condition) every working day for four weeks. Primary outcome measure is the change of tinnitus distress as quantified by the Tinnitus Questionnaire (TQ). Secondary outcome measures are tinnitus loudness and annoyance as well as tinnitus change during and after treatment. Audiologic and speech audiometric measurements will be performed to assess potential side effects. The aim of the present trail is to investigate effectiveness and safety of a four weeks cTBS treatment on chronic tinnitus and to compare two areas of stimulation. The results will contribute to clarify the therapeutic capacity of rTMS in tinnitus.
\end{abstract}

Trial registration: The trial was registered with the clinical trials register of http:// www.clinicaltrials.gov (NCT005 I8024). 


\section{Background Tinnitus}

Tinnitus is the perception of sounds or noise in the absence of an external stimulus. About 10 to $15 \%$ of the general population [1] report this auditory phantom perception [2]. Around 1 to $2 \%$ of the patients are seriously impaired [1]. Sleep disturbances, depression, irritability, and anxiety symptoms are the most common psychiatric comorbidities of tinnitus [1,3]. Current therapies focus on the "management of tinnitus", i. e. they are a means to reduce tinnitus perception or awareness, and treating comorbidities rather than curing tinnitus itself [4-6]. Cognitive-behavioural therapies yield some relief [7], but are often not sufficient. To date, there is no pharmacological intervention or device available that has been proven to reliably reduce tinnitus. The pathophysiological causes of tinnitus are poorly understood which impedes the development of rational and evidence based therapies. However, recent findings indicate that chronic tinnitus is the result of maladaptive reorganization in the central auditory system [8] that can be reflected in hyperacitivity of cortical areas involved in the perception and processing of auditory information [6,9-14].

\section{Transcranial magnetic stimulation}

Transcranial magnetic stimulation (TMS) is a non invasive method to depolarize cortical neurons based on the principle of electromagnetic induction [15]. Applying series of rapid consecutive single stimuli is called repetitive TMS (rTMS). Low frequency rTMS $(1 \mathrm{~Hz})$ has been shown to decrease the excitability of the motor cortex [16], whereas high frequency rTMS $(\geq 5 \mathrm{~Hz}$ ) produces the opposite effect [17]. rTMS causes activity changes in regions interconnected with the stimulated area through mono- or polysynaptic connections [18], modulates subcortical transmitter concentrations [19], and was shown to induce morphological modifications in stimulated areas as well as in areas linked to them [20]. Based on these findings, rTMS is used experimentally to treat a wide range of clinical disorders that may involve altered states of cortical excitability [21], such as major depression [22,23], auditory hallucinations [24], and stroke [25].

\section{TMS and tinnitus}

Repetitive transcranial magnetic stimulation has recently been adopted to strengthen the concept of focally increased cortical excitability as a pathophysiological mechanism of tinnitus perception. Initially, it was shown that a high-frequency $(10 \mathrm{~Hz})$ rTMS induced 'virtual' lesion of temporoparietal cortical areas can transiently reduce tinnitus $[26,27]$. Further studies indicated that the effect size was negatively correlated with the tinnitus duration $[28,29]$. On the basis of these data, it has been proposed that a rTMS-induced reduction of hyperactivity of these cortical areas could yield beneficial effects for patients with chronic tinnitus [27]. To test this notion, low frequency rTMS was applied to areas of the temporoparietal cortex showing tinnitus-related hyperacitivity. Immediately after this intervention, tinnitus loudness was reduced for up to $30 \mathrm{~min}$. The degree of reduction was dose dependent, and negatively correlated with tinnitus duration [11]. Several small clinical studies indicate that repeated application of low-frequency rTMS up to 2 weeks may have lasting ameliorating effects on chronic tinnitus [30-37]. However, the effect size was mostly moderate and interindividual responses as well as effect duration were highly variable. Further studies are needed to assess the clinical relevance of rTMS treatment in tinnitus and to identify the optimal stimulation paradigms as well as the most effective stimulation target site. Recently, continuous theta burst stimulation (cTBS) has been put forward as a modified rTMS paradigm [38] that reduces cortical excitability by applying three pulses at $50 \mathrm{~Hz}$ repeated every $200 \mathrm{~ms}$ over $40 \mathrm{~s}[38,39]$ at an intensity of $80 \%$ active motor threshold (AMT). For clinical purposes cTBS appears to be an applicable technique due to its low intensity, short duration, and similar efficacy [40]. In a first case report we demonstrated that cTBS is an effective approach to treat tinnitus [41].

\section{Objectives}

We hypothesize that compared to sham stimulation cTBS can induce a distinctive attenuation of tinnitus distress and loudness and thus be of therapeutic value. Further aims of the study are:

- To compare the effect of cTBS on the secondary auditory cortex with its effect on the temporoparietal association cortex.

- Assessment of safety in terms of impairment in audiologic and speech-audiometric measures as well as the documentation of other unwanted side effects.

\section{Design and Methods}

This randomised, placebo controlled study consists of 3 arms in a parallel design (Figure 1). Tinnitus distress, loudness and annoyance will be assessed before, during, and after 4 weeks of bilateral cTBS. Forty-eight patients will be randomised in an 1:1:1 ratio to either cTBS over the secondary auditory cortex (SAC), the temporoparietal association cortex (TAC) or sham stimulation (placebo: PLC).

\section{Population}

The patient population will consist of patients with a wide middle range of tinnitus duration and excluding those with an increased risk for adverse effect of TBS. 


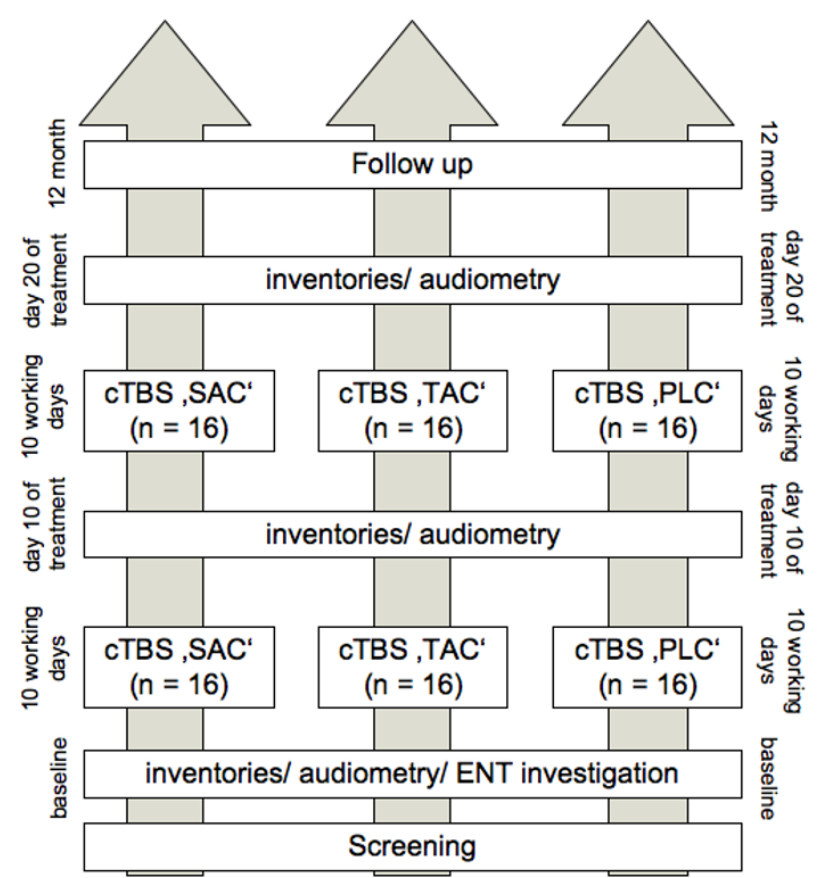

Figure I

Study Design.

Inclusion criteria

- Written informed consent

- Age: between 18 and 75 years

- Chronic tinnitus since at least 6 month, but not more than 5 years.

\section{Exclusion criteria}

- Objective tinnitus

- Acute or chronic inflammation of the middle ear, acute hearing loss, hearing loss due to an acoustic trauma within less than past 6 weeks

- Morbus Menière or fluctuating hearing loss

- Epilepsy

- Brain trauma

- Brain surgery

- Heart pacemaker

- Intake of anticonvulsants, antipsychotics, and regular intake of benzodiazepin

\section{- Suicidality}

- Other severe pathological chronic condition that might confound treatment effects or interpretation of data

- Pregnancy

- Participation in another clinical study regarding tinnitus within the past 3 months and during enrolment in our study.

\section{Data sets}

For evaluation of security all data gathered will be used and analyzed as treated. The analysis of the efficacy measures, especially with respect to the stimulus placement, requires a concentration on patients treated per protocol (PP). Missing observations are imputed using a full conditional Markov-Chain Monte Carlo method for variables with more than $75 \%$ valid values. An exploratory analysis of all dependent variables by the randomised treatment, $\mathrm{i}$. e. the mere intention to treat (ITT), will be conducted to plan subsequent confirmatory studies.

\section{Screening}

Patients will be recruited via announcement in the local press, the tinnitus outpatient clinic at the Department of Psychiatry and Psychotherapy, and the outpatient clinic of the Department of Otorhinolaryngology of the University Hospital of Tübingen. A psychiatrist and an otolaryngologist will assess the eligibility according to the inclusion and exclusion criteria listed above.

\section{Baseline assessments}

The detailed time course and plan of the study is displayed in Table 1.

- Ear Nose and Throat (ENT) examination and audiological assessment including

- Standard pure tone audiometry

- Speech audiometry in quiet (mono- and multisyllables, "Freiburger speech test")

- Hearing in noise test ("Oldenburger sentence test") [42]

- Audiological tinnitus matching

- Structered Tinnitus Interview (STI) [43]

- Tinnitus Questionnaire (TQ) [44,45] 
Table I: Time course of the study

\begin{tabular}{|c|c|c|c|c|c|c|c|c|c|c|c|}
\hline \multirow{3}{*}{$\begin{array}{l}\text { Investigation } \\
\text { Visit \# }\end{array}$} & \multirow{3}{*}{$\begin{array}{l}\text { Screening } \\
\text { vo }\end{array}$} & \multirow{3}{*}{$\begin{array}{l}\text { Baseline } \\
\text { VI }\end{array}$} & \multicolumn{4}{|c|}{ Treatment } & \multirow{2}{*}{\multicolumn{5}{|c|}{$\begin{array}{l}\text { Follow-Up } \\
11 \text { months }\end{array}$}} \\
\hline & & & \multicolumn{2}{|c|}{2 weeks } & \multicolumn{2}{|c|}{2 weeks } & & & & & \\
\hline & & & $\mathrm{V} 2-10$ & VII & VI2-20 & V2I & V22 & V23 & V24 & V25 & $\mathrm{V} 26$ \\
\hline informed consent & $x$ & & & & & & & & & & \\
\hline demograph. data & $x$ & & & & & & & & & & \\
\hline inclusion/exclusion criteria & $x$ & & & & & & & & & & \\
\hline randomization & & $x$ & & & & & & & & & \\
\hline ENT investigation/tinnitus localization & & $x$ & & & & & & & & & \\
\hline audiogram/speech audiometry & & $x$ & & $x$ & & $x$ & & & & & \\
\hline STI & & $x$ & & & & & & & & & \\
\hline cTBS treatment SAC, TAC, PLC & & & $x$ & $x$ & $x$ & $x$ & & & & & \\
\hline documentation of side effects & & & $x$ & $x$ & $x$ & $x$ & & & & & \\
\hline TQ & & $x$ & & $x$ & & $x^{*}$ & $x$ & $x$ & $x$ & $x$ & $x$ \\
\hline VAS (distress, loudness) & & $x$ & $x$ & $x$ & $x$ & $x$ & $x$ & $x$ & $x$ & $x$ & $x$ \\
\hline VAS (change) & & & & $x$ & & $x$ & & & & & \\
\hline SCL-90R & & $x$ & & $x$ & & $x$ & $x$ & $x$ & $x$ & $x$ & $x$ \\
\hline BDI & & $x$ & & $x$ & & $x$ & $x$ & $x$ & $x$ & $x$ & $x$ \\
\hline
\end{tabular}

*primary outcome measure

- Visual Analogue Scales (VAS) for tinnitus loudness and annoyance [46]

- Beck Depression Inventory (BDI) [47]

- Symptom checklist 90-revised (SCL-90R) [48].

\section{Safety}

Safety measures are pure tone and speech audiometry as well as speech understanding in noise tests (at Baseline, after 2 weeks of treatment and after the end of the treatment). Adverse events are assessed daily and documented according to the GCP guidelines. Safety and adverse events will be reported together with the results. The occurence of possible adverse effects will be reported in a case report form and handled in compliance with GCP. Study monitoring is provided by CenTrial (Tübingen, Germany).

\section{Outcome measures}

The primary efficacy measure in the present study is the change of the tinnitus distress as measured by the TQ [45] after 4 weeks of cTBS. The TQ [45]), originally developed by Hallam et al. [49], is the most widely used, well validated and reliable inventory [50] to quantify tinnitus ditress available in German language [30,32,34,41,5158]. It comprises 52 multi-scaled items in order to assess emotional and cognitive handicaps, penetrance of the tinnitus, hearing problems, sleep disturbances, and somatic discomfort. The TQ was used in pharmacological, behavioural and TMS studies. Pharmacological studies did not yield therapeutic effects [58,59]. Behavioural therapy yield effect sizes between 6 and $28 \%$ on the TQ $[60,61]$. Pilot rTMS studies reported effects sizes between 5 and $25 \%[34,41,52]$. 
Secondary efficacy variables are tinnitus change [46] and the changes in BDI [47] and in the VAS for tinnitus loudness and tinnitus annoyance $[46,62]$.

Changes regarding psychopathology will be measured using the SCL-90R [48]. Feasibility of the study will be assessed and reported together with the results.

\section{Follow-up assessments}

Duration of treatment effects will be measured (TQ, VAS, BDI) $6,8,16,28$, and 56 weeks after the first stimulation. Furthermore, 6 months after the end of treatment patients will be asked to report if they regard the estimated benefit as worth the effort.

\section{Withdrawal from the study}

In case of endangerment of personal security or lack of compliance or withdrawal of informed consent, a patient will instantly be excluded from further participation in the study.

\section{Sample size calculation}

Effect size and dispersion were taken from our last study of TMS against tinnitus [34]. The ratios of tinnitus distress score after treatment to tinnitus distress score at baseline are assumed to follow normal distributions with standard deviation 0.18 after taking logarithms. A relevant effect size of 0.19 logarithmic units would arise from the ratio of geometric mean scores of $80 \%$ of baseline after efficacious stimulation to $97 \%$ after sham stimulation. All three pairwise group comparisons will be conducted. Therefore the probabilities of the errors of first and second kind are Bonferroni-adjusted to significance level 0.017 and power 0.93 locally to ensure multiple level 0.05 and multiple power 0.8 . Consequently the planned number of patients to be included in the study is 48 , i. e. 16 in each arm. A closed testing procedure preserves the multiple significance level and power while treatments are compared at different points in time.

\section{Data analysis}

The geometric mean tinnitus score (TQ) in percent baseline will be compared between the three groups after the full treatment course of 4 weeks after the first stimulation, after 6 weeks, as well as 28 and 56 weeks after the first CTBS treatment in that prespecified order, as we expect effects sizes to have that order. If one group comparison is not statistically significant, the following comparisons are rated as not significant too. The confidence intervals for ratios of geometric means of tinnitus scores at 4 and 6 weeks will be computed from an analysis of variance (ANOVA) with factors group (SAC, TAC, PLC) and time (4 and 6 weeks) and the group*time interaction. Further ANOVAs of TQ with the between subject factor group will be carried out after 8,28 , and 56 weeks after the first treat- ment. Their residual variances are expected to differ. The longitudinal course of the stimulation effect will be described using all TQ measurements, including measurements at 2 and 16 weeks. Secondary efficacy measures will be analysed similarly. Only the tinnitus change cannot be divided by a baseline value and will be analysed by ordinal logistic regression as it is ordinal. The daily measurements on visual analog scales will be shown in diagrams. Confidence interval computations will assume normality of logit-transformed VAS. The routinely sampled safety data will all be plotted for each patient and will be used to quantify intensity and duration of adverse effects should such occur. Data analysis will be performed in the Department of Medical Biometry of University Hospital of Tübingen using JMP 7.0 (SAS Institute Inc.) after imputation with SPSS 17.0 (SPSS Inc.).

\section{Randomization}

A randomisation list was prepared by the Department of Medical Biometry of University Hospital of Tübingen (RV) using permuted blocks. Allocations were concealed in opaque sealed envelopes, that are opened by a third person immediately prior to the first treatment.

\section{Treatment}

After screening, written informed consent, physical ENT examination and audiological assessments, patients will be assigned to one of the real (SAC, TAC) or sham (PLC) treatment groups. TMS will be applied using a Magstim Super Rapid (The Magstim Company Ltd, Whitland, UK) with a figure-eight coil (diameter of each winding: 70 $\mathrm{mm}$, biphasic stimulus of $250 \mu \mathrm{s}$, peak magnetic field: 2T). The individual motor threshold (AMT) will be assessed at the beginning of the first treatment session. AMT is defined as minimum stimulation intensity required to induce a motor evoked potential (MEP) of more than $200 \mu \mathrm{V}$ on at least 5 out of 10 trials from the contralateral abductor pollicis brevis muscle (APB) while maintaining a voluntary contraction of about $20 \%$ of maximum [39]. Stimulation (CTBS) intensity is $80 \%$ AMT and will be applied to each side for $40 \mathrm{~s}$ in alternating order. Fifteen minutes after the first two trains, a second pair of cTBS will be applied (a total of 2400 stimuli per day). Patients receive cTBS treatment each working day for 4 weeks.

\section{Blinding}

The endpoint assessor remains masked to the treatment until the final data analysis. For adequate masking of the patients, sham stimulations will be performed at the lower occiput in the same distance to the ear $[11,27,63]$. In this way, the control stimulation is accompanied by a similar noise (between 60 and $75 \mathrm{~dB}$ ) and comparable aversive sensation (pricking, muscle twitches). 


\section{Data Management}

Data are collected on paper case report forms that are stored in a safe place until 10 years after completion of the trial. Data are entered directly in the statistical analysis software file on a safely kept computer with individual user passwords. This process is replicated by a second person and the resulting files are compared regularly, so that ambiguous entries can be questioned in short time. Plausibility checks, source data verification and quality control are carried out by an external monitor.

\section{Ethics, Consent, Study Organization and Registration}

The trial will be conducted in agreement with the principles of the Declaration of Helsinki, and with the guidlines of Good Clinical Practice (GCP) of the International Conference on Harmonisation of Technical Requirements for Registration of Pharmaceuticals for Human Use (ICH). The protocol was approved of by the local Indpendent Ethics Committee (Institutional Review Board). Funding is provided by the German Research Council. The investigator will explain the benefits and risks of participation in the study to each subject and will provide an informed consent form approved by the independent ethics committee. Only patients, who sign the form, will be included in the study. Results will be published so that patients cannot be identified. All eligible patients are seen by a psychiatrist and an otolaryngologist and are enrolled after giving informed consent. All findings will be recorded in the patients' medical records and the CRF provided for this study. Study auditing, CRF compilation and study monitoring is performed by CenTrial GmbH (Tübingen, Germany).

\section{Discussion}

The available data on the efficacy of tinnitus treatment with rTMS is incomplete [30-37]. The effect size, the clinical relevance and the optimal stimulation parameters are virtually unknown. This placebo-controlled phase II clinical trial has been designed to investigate the efficacy of a 4 weeks treatment with bilateral cTBS on chronic tinnitus and to compare the effectiveness of two different stimulation areas. Since the laterality of tinnitus-related cortical hyperactivity and rTMS effects in mono- and bilateral tinnitus have been shown to be interindividually variable $[11,64]$, we opted for a bilateral cTBS. Recent studies on therapeutic efficacy of rTMS in major depression pointing towards a superior effectiveness of longer treatment durations [23] prompted us to extend cTBS treatment to 4 weeks. Moreover, against the background of a dose dependency of rTMS effects in tinnitus [11] and in order to assure a sufficient dose of stimulation, the patients will receive two stimulation trains on each side. Hence, bilateral, long-term application of cTBS to two different cortical sites will provide comprehensive data for an evaluation of the clinical relevance of TMS treatment in chronic tinnitus and an improvement of treatment parameters. Based on the effect size and standard deviation of the Intention-to-Treat analysis as well as on the finding of the optimal stimulation localization a larger clinical efficacy study will be designed.

\section{Abbreviations}

APB: abductor pollicis brevis muscle; AMT: active motor threshold; BDI: Beck Depression Inventory; ITT: intention to treat; LOCF: last observation carried forward; MEP: motor evoked potential; MT: motor treshold; PLC: placebo (sham stimulation); SAC: secondary auditory cortex; SCL-90R: Symptom Checklist 90-revised; TAC: tertiary auditory cortex; TQ: Tinnitus Questionnaire; VAS: Visual Analogue Scale

\section{Competing interests}

The authors declare that they have no competing interests.

\section{Authors' contributions}

All authors contributed to the study design. CP is the initiator and principal investigator of the study. RV is the trial statistician, and carried out the randomisation and sample size calculations. CA is the trial manager. CP, CA, and SP drafted the original study protocol. The manuscript was written by CP, CA, and RV. All authors have read and approved the final manuscript.

\section{Acknowledgements}

The study is funded by the German Research Council (DFG; 253 PLI-I).

\section{References}

I. Dobie RA: Depression and tinnitus. Otolaryngol Clin North Am 2003, 36:383-388.

2. Jastreboff PJ: Phantom auditory perception (tinnitus): mechanisms of generation and perception. Neurosci Res 1990, 8:221-254.

3. Schaaf H, Dolberg D, Seling B, Martner M: [Comorbidity of tinnitus and psychiatric disorders]. Nervenarzt 2003, 74:72-75.

4. Dobie RA: A review of randomized clinical trials in tinnitus. Laryngoscope 1999, 109:1202-1211.

5. Goodey R: Tinnitus treatment - state of the art. Prog Brain Res 2007, 166:237-246. [Review].

6. Henry J, Dennis K, Schechter M: General review of tinnitus: Prevalence, mechanisms, effects, and management. J Speech Lang Hear Res 2005, 48: I204-1235. [Review].

7. Martinez-Devesa P, Waddell A, Perera R, M T: Cognitive behavioural therapy for tinnitus. Cochrane Database Syst Rev 2007:CD005233. [Review].

8. Mühlnickel W, Elbert T, Taub E, Flor $\mathrm{H}$ : Reorganization of auditory cortex in tinnitus. Proc Natl Acad Sci USA 1998, 95: 10340-10343.

9. Eggermont JJ, Kenmochi M: Salicylate and quinine selectively increase spontaneous firing rates in secondary auditory cortex. Hear Res 1998, I I7(I-2): 149-160.

10. Lockwood AH, Wack DS, Burkard RF, Coad ML, Reyes SA, Arnold SA, Salvi RJ: The functional anatomy of gaze-evoked tinnitus and sustained lateral gaze. Neurology 200I, 56:472-480.

II. Plewnia C, Reimold M, Najib A, Brehm B, Reischl G, Plontke S, Gerloff $C$ : Dose-dependent attenuation of auditory phantom perception (tinnitus) by PET-guided repetitive transcranial magnetic stimulation. Hum Brain Mapp 2007, 28(3):238-246.

12. Rauschecker J: Auditory cortical plasticity: A comparison with other sensory systems. Trends Neurosci 1999, 22(2):74-80. 
13. Salvi R, Wang J, Ding D: Auditory plasticity and hyperactivity following cochlear damage. Hear Res 2000, I47(I-2):26I-274. [Review]

14. Weisz N, Moratti S, Meinzer M, Dohrmann K, Elbert T: Tinnitus perception and distress is related to abnormal spontaneous brain activity as measured by magnetoencephalography. PLoS Med 2005, 2(6):el 53.

15. George MS, Nahas Z, Kozel FA, Goldman J, Molloy M, Oliver N: Improvement of depression following transcranial magnetic stimulation. Curr Psychiatry Rep 1999, I(2): I |4-I 24.

16. Chen R, J C, Gerloff C, Celnik P, Wassermann EM, Hallett M, Cohen L: Depression of motor cortex excitability by low-frequency transcranial magnetic stimulation. Neurology 1997 48: $1398-1403$

17. Berardelli A, Inghilleri M, Rothwell JC, Romeo S, Curra A, Gilio F, Modugno N, Manfredi M: Facilitation of muscle evoked responses after repetitive cortical stimulation in man. Exp Brain Res 1998, I 22:79-84.

18. Hayashi T, Ohnishi T, Okabe S, Teramoto N, Nonaka Y, Watabe H, Imabayashi E, Ohta $\mathrm{Y}$, Jino H, Ejima N, Sawada T, lida $\mathrm{H}$, Matsuda $\mathrm{H}$, Ugawa $Y$ : Long-term effect of motor cortical repetitive transcranial magnetic stimulation [correction]. Ann Neurol 2004, 56:77-85. [Erratum in: Annals of Neurology 56(2): 3II].

19. Strafella AP, Paus T, Fraraccio M, Dagher A: Striatal dopamine release induced by repetitive transcranial magnetic stimulation of the human motor cortex. Brain 2003, I 26(Pt I 2):2609-26I5.

20. May A, Hajak G, Ganssbauer S, Steffens T, Langguth BT, Eichhammer $P$ : Structural Brain Alterations following 5 Days of Intervention: Dynamic Aspects of Neuroplasticity. Cereb Cortex 2007, 17:205-210.

21. Hoffman R, Cavus I: Slow transcranial magnetic stimulation, long-term depotentiation, and brain hyperexcitability disorders. Am J Psychiatry 2002, I59(7): 1093-I I02. [Review]

22. Herwig U, Fallgatter A, Höppner J, Eschweiler G, Kron M, Hajak G, Padberg F, Naderi-Heiden A, Abler B, Eichhammer P, Grossheinrich N, Hay B, Kammer T, Langguth B, Laske C, Plewnia C, Richter M, Schulz M, Unterecker S, Zinke A, Spitzer M, Schönfeldt-Lecuona C: Antidepressant effects of augmentative transcranial magnetic stimulation: randomised multicentre trial. $\mathrm{Br} J$ Psychiatry 2007, 191:44|-448.

23. O'Reardon J, Solvason H, Janicak P, Sampson S, Isenberg K, Nahas Z, McDonald W, Avery D, Fitzgerald P, Loo C, Demitrack M, George M, Sackeim H: Efficacy and safety of transcranial magnetic stimulation in the acute treatment of major depression: a multisite randomized controlled trial. Biol Psychiatry 2007, 62(II): $1208-1216$.

24. Aleman A, Sommer I, Kahn R: Efficacy of slow repetitive transcranial magnetic stimulation in the treatment of resistant auditory hallucinations in schizophrenia: a meta-analysis. J Clin Psychiatry 2007, 68(3):4I6-42I.

25. Talelli P, Greenwood R, Rothwell J: Exploring Theta Burst Stimulation as an intervention to improve motor recovery in chronic stroke. Clin Neurophysiol 2007, I I 8(2):333-342.

26. Plewnia C, Bartels M, Gerloff C: Cortical Mapping of Auditory Phantom Perception (Tinnitus). A Pilot Study. Akt Neurol 2000, 27:S203

27. Plewnia C, Bartels M, Gerloff C: Transient suppression of tinnitus by transcranial magnetic stimulation. Ann Neurol 2003 53:263-266.

28. de Ridder D, Verstraeten E, Kelen K van der, de Mulder G, Sunaert $S$, Verlooy J, Heyning $P$ van de, Moller A: Transcranial magnetic stimulation for tinnitus: influence of tinnitus duration on stimulation parameter choice and maximal tinnitus suppression. Otol Neurotol 2005, 26:616-619.

29. Fregni F, Marcondes R, Boggio PS, Marcolin MA, Rigonatti SP, Sanchez TG, Nitsche MA, Pascual-Leone A: Transient tinnitus suppression induced by repetitive transcranial magnetic stimulation and transcranial direct current stimulation. Eur J Neurol 2006 , |3(9):996-100|.

30. Eichhammer P, Langguth B, Marienhagen J, Kleinjung T, Hajak G: Neuronavigated repetitive transcranial magnetic stimulation in patients with tinnitus: A short case series. Biol Psychiatry 2003, 54:862-865.

31. Langguth B, Eichhammer P, Zowe M, Marienhagen J, Kleinjung T, Jacob $P$, Sand $P, G$ GH: [Low frequency repetitive transcranial magnetic stimulation (rTMS) for the treatment of chronic tinnitus- are there long-term effects?]. Psychiatr Prax (Suppl I) 2004, $31:$ :S52-54. [German].

32. Kleinjung $T$, Eichhammer $P$, Langguth $B$, Jacob $P$, Marienhagen J, Hajak $G$, Wolf SR, Strutz J: Long-term effects of repetitive transcranial magnetic stimulation (rTMS) in patients with chronic tinnitus. Otolaryngol Head Neck Surg 2005, 1 32:566-569.

33. Langguth B, Hajak G, Kleinjung T, Pridmore S, Sand P, Eichhammer P: Repetitive transcranial magnetic stimulation and chronic tinnitus. Acta Otolaryngol Suppl 2006, 556: I02-105. [Review]

34. Plewnia C, Reimold M, Najib A, Reischl G, Plontke SK, Gerloff C: Moderate therapeutic efficacy of PET-navigated transcranial magnetic stimulation against chronic tinnitus: a randomised, controlled pilot study. J Neurol Neurosurg Psychiatry 2007, 78(2): I52-I56.

35. Kleinjung $T$, Steffens $T$, Londero A, Langguth B: Transcranial magnetic stimulation (TMS) for treatment of chronic tinnitus: clinical effects. Prog Brain Res 2007, 166:359-367. [Review].

36. Smith J, Mennemeier M, Bartel T, Chelette K, Kimbrell T, Triggs W, Dornhoffer J: Repetitive transcranial magnetic stimulation for tinnitus: a pilot study. Laryngoscope 2007, I I 7(3):529-534

37. Khedr E, Rothwell J, Ahmed M, El-Atar A: Effect of daily repetitive transcranial magnetic stimulation for treatment of tinnitus: comparison of different stimulus frequencies. I Neurol Neurosurg Psychiatry 2008, 79(2):212-2I 5.

38. Huang YZ, Rothwell JC: The effect of short-duration bursts of high-frequency, low-intensity transcranial magnetic stimulation on the human motor cortex. Clin Neurophysiol 2004, I 15:1069-1075.

39. Huang YZ, Edwards MJ, Rounis E, Kailash PB, Rothwell JC: Theta burst stimulation of the human motor cortex. Neuron 2005 , 45:20I-206.

40. Zafar N, Paulus W, Sommer M: Comparative assessment of best conventional with best theta burst repetitive transcranial magnetic stimulation protocols on human motor cortex excitability. Clin Neurophysiol 2008, I I 9(6): 1393-1399.

4I. Soekadar S, Arfeller C, Rilk A, Plontke S, Plewnia C: Continous TBS in the treatment of incapacitating tinnitus accompanied by symptoms of severe depression. CNS Spectr 2009, I4(4): I56-I59.

42. Wagener K, Kollmeier B: Göttinger und Oldenburger Satztest. Z Audiol 2004, 43: |34-|4|.

43. Hiller W, Goebel G: Assessing audiological, pathophysiological, and psychological variables in chronic tinnitus: a study of reliability and search for prognostic factors. Int J Behav Med 1999. 6(4):3।2-330.

44. Hiller W, Goebel G, Rief W: Reliability of self-rated tinnitus distress and association with psychological symptom patterns. BrJ Clin Psychol 1994, 33(Pt 2):23I-239.

45. Goebel G, Hiller W: [The tinnitus questionnaire: A standard instrument for grading the degree of tinnitus. Results of a multicenter study with the tinnitus questionnaire]. HNO 1994, 42:166-I72.

46. Zenner $\mathrm{H}$, de Maddalena $\mathrm{H}$, Zalaman I: Validity and reliability study of three tinnitus self-assessment scales: loudness, annoyance and change. Acta Otolaryngol 2005, I 25(II): I I84-II 88 .

47. Hautzinger M, Bailer M, Worall H, Keller F: Beck Depressions Inventar (BDI). In Testhandbuch (2. Auflage) Bern: Hans Huber; 1995.

48. Derogatis LR: SCL-90-R: Administration scoring and procedures manual-I Johns Hopkins University School of Medicine, Baltimore: Clinical Psychometric Research; 1977

49. Hallam R, SC J, Hinchcliff R: Cognitive variables in tinnitus annoyance. BJ Clin Psychol 1988, 27:213-222.

50. Meikle M, Stewart B, Griest S, Martin W, Henry J, Abrams H, McArdle R, Newman C, Sandridge S: Assessment of tinnitus: measurement of treatment outcomes. Prog Brain Res 2007, I66:5 I I-52 I. [Review].

51. deRidder D, Loo E van der, Kelen K van der, Menovsky T, Heyning P van de, A M: Do tonic and burst TMS modulate the lemniscal and extralemniscal system differential? Int J Med Sci 2007, 4(5):242-246.

52. Kleinjung $T$, Steffens $T$, Londero $A$, Langguth $B$ : Which tinnitus patients benefit from Transcranial magnetic stimulation? Otolaryngol Head Neck Surg 2007, 137:589-595. 
53. Landgrebe $M$, Binder $\mathrm{H}$, Koller $M$, Eberl $\mathrm{Y}$, Kleinjung $\mathrm{T}$, Eichhammer P, Graf E, Hajak G, Langguth B: Design of a placebo-controlled randomized study of the efficacy of repetitive transcranial magnetic stimulation for the treatment of chronic tinntius. BMC Psychiatry 2008, 8:23.

54. Langguth B, Eichhammer P, Wiegand R, Marienhegen J, Maenner P, Jacob P, Hajak G: Neuronavigated rTMS in a patient with chronic tinnitus. Effects of $\mathbf{4}$ weeks treatment. Neuroreport 2003, I 4(7):977-980.

55. Langguth $B$, Eichhammer $P$, Zowe $M$, Kleinjung $T$, Jacob $P$, Binder $H$, Sand P, Hajak G: Altered motor cortex excitability in tinnitus patients: a hint at crossmodal plasticity. Neurosci Lett 2005 380(3):326-329.

56. Langguth $B$, Eichhammer $P$, Kreutzer A, Maenner $P$, Marienhagen J, Kleinjung $T$, Sand $P$, Hajak G: The impact of auditory cortex activity on characterizing and treating patients with chronic tinnitus- first results from a PET study. Acta Otolaryngol Suppl 2006:84-88.

57. Langguth B, Zowe M, Landgrebe M, Sand P, Kleinjung T, Binder H, Hajak G, Eichhammer $P$ : Transcranial magnetic stimulation for the treatment of tinnitus: a new coil positioning method and first results. Brain Topogr 2006, 18(4):24I-247.

58. Salembier L, De Ridder $D$, Heyning P Van de: The use of flupirtine in treatment of tinnitus. Acta Otolaryngol Suppl 2006, 556:93-95.

59. Mazurek B, Haupt H, Szczepek A, Sandmann J, Gross J, Klapp B, Kiesewetter H, Kalus U, Stöver T, Caffier P: Evaluation of vardenafil for the treatment of subjective tinnitus: a controlled pilot study. J Negat Results Biomed 2009, 8:3.

60. Rief W, Weise C, Kley N, Martin A: Psychophysiological treatment of chronic tinnitus: A randomized clinical trial. Psychosomatic Medicine 2005, 67:833-838.

61. Caffier P, Haupt H, Schere H, Mazurek B: Outcomes of long-term outpatient tinnitus coping therapy: psychometric changes and value of tinnitus-control instruments. Ear and Hearing 2006, 27:619-627.

62. Hiller W, Goebel G: When tinnitus loudness and annoyance are discrepant: audiological characteristics and psychological profile. Audiol Neurootol 2007, I 2(6):39|-400.

63. Plewnia C, Kammer T, Gerloff $C$ : Treating tinnitus with transcranial magnetic stimulation. Biol Psychiatry 2004, 55: I I 7. [Comment].

64. Folmer R: Lateralization of neural activity associated with tinnitus. Neuroradiology 2007, 49(8):689-691.

Publish with Bio Med Central and every scientist can read your work free of charge

"BioMed Central will be the most significant development for disseminating the results of biomedical research in our lifetime. "

Sir Paul Nurse, Cancer Research UK

Your research papers will be:

- available free of charge to the entire biomedical community

- peer reviewed and published immediately upon acceptance

- cited in PubMed and archived on PubMed Central

- yours - you keep the copyright
BioMedcentral 